\title{
Joint Power Allocation and Splitting Control in SWIPT-Aided Multi-Carrier NOMA System
}

\author{
Jie Tang ${ }^{1}$, Jingci Luo ${ }^{1}$, Junhui $\mathrm{Ou}^{1}$, Xiuyin Zhang ${ }^{1}$, Nan $\mathrm{Zhao}^{2}$, Danial So ${ }^{3}$, \\ and Kai-Kit Wong ${ }^{4}$ \\ 1 South China University of Technology, Guangzhou, China \\ eejtang@scut.edu.cn; 201720110615@mail.scut.edu.cn; \\ 2 Dalian University of Technology, Dalian, China \\ zhaonan@dlut.edu.cn \\ 3 The University of Manchester, Manchester, UK \\ d.so@manchester.ar.uk \\ 4 University College London, London, UK \\ kai-kit.wong@ucl.ac.uk
}

\begin{abstract}
The combination of non-orthogonal multiple access (NOMA) and simultaneous wireless information and power transfer (SWIPT) contributes to improve the spectral efficiency (SE) and the energy efficiency (EE) at the same time. In this paper, we investigate the throughput maximization problem for the downlink multi-carrier NOMA (MC-NOMA) system with the application of power splitting (PS)-based SWIPT, in which power allocation and splitting are jointly optimized with the constraints of maximum transmit power supply as well as the minimum demand for energy harvesting (EH). To tackle the non-convex problem, a dual-layer approach is developed, in which the power allocation and splitting control are separated and the corresponding sub-problems are respectively solved through Lagrangian duality method. Simulation results validate the theoretical findings and demonstrate the superiority of the application of PS-based SWIPT to MC-NOMA over SWIPT-aided single-carrier NOMA (SC-NOMA) and SWIPT-aided orthogonal multiple access (OMA).
\end{abstract}

Keywords: Multi-carrier non-orthogonal multiple access (MC-NOMA), simultaneous wireless information and power transfer (SWIPT), deep learning.

\section{Introduction}

With the rapid development of fifth generation (5G) and its advanced application scenarios, the limited spectrum and energy resources are increasingly difficult to meet the requirements for the communication system. Hence, it is considerably significant to improve the spectrum efficiency (SE) and energy efficiency (EE). The non-orthogonal multiple access (NOMA) scheme has been 
considered as a significant technique to achieve a higher SE for 5G and the future communication system due to the elimination of channel orthogonality [1]. Besides, NOMA technology enables the communication system to provide higher data rate, lower latency, greater reliability and larger connectivity, etc. [2]. Thus, NOMA has aroused great attention and the application of NOMA to other advanced techniques has also been investigated, including multiple-input multipleoutput (MIMO) [3], cognitive radio [4], multi-point cooperative relaying [5], etc.. On the other hand, simultaneous wireless information and power transfer (SWIPT) [6], which makes it possible to collect energy and receive information parallelly, is viewed as an energy-efficient solution to the green communication system. Therefore, it has attracted extensive concern in both academic and industry.

Previous studies in $[7,8]$ have investigated the performance comparison between NOMA and the conventional orthogonal multiple access (OMA) with the application of SWIPT. However, most of the existing works considered the singlecarrier NOMA (SC-NOMA) systems and the performance of SWIPT-aided MCNOMA is still an open topic. Motivated by this conversation, we considered a novel system which combines the spectrum-efficient MC-NOMA and the energyefficient SWIPT, where the total throughput maximization problem is investigated with the constraints of transmit power supply and energy harvesting $(\mathrm{EH})$ requirement.

\section{System Model and Problem Formulation}

\subsection{System Model}

In this section, we focus on the downlink of MC-NOMA system with the application of PS-based SWIPT, in which one BS communicates with $K$ MUs via $N$ subcarriers (SCs). Denote the set of all MUs' indexes and the set of all SCs' indexes as $\mathcal{K}=\{1,2, \cdots, K\}$ and $\mathcal{N}=\{1,2, \cdots, N\}$, respectively. The available bandwidth $B W$ is equally divided into $N$ orthogonal SCs and hence the bandwidth of each SC is $B W_{n}=B W / N$. Thus, the received signal of the $k$-th MU via the $n$-th $\mathrm{SC}$ is given by

$$
y_{n, k}=h_{n, k}\left(\sqrt{p_{n, k}} s_{n, k}+\sum_{j \in \mathcal{K}, j \neq k} \sqrt{p_{n, j}} s_{n, j}\right)+z_{n, k},
$$

where $h_{n, k}$ represents the channel coefficient from the BS to the $k$-th MU over the $n$-th SC; $s_{n, k}\left(s_{n, j}\right)$ indicates the data symbol transmitted from the BS to the $k$-th $(j$-th) MU over the $n$-th SC, which is a random signal with the energy of $\mathbb{E}\left[\left|s_{n, k}\right|^{2}\right]\left(\mathbb{E}\left[\left|s_{n, k}\right|^{2}\right]\right)=1 ; z_{n, k} \sim \mathcal{C N}\left(0, \sigma_{n, k}^{2}\right)$ denotes the additive white Gaussian noise (AWGN) to the $k$-th MU on the $n$-th SC.

At the receiving end, the received signal of the $k$-th $(k \in \mathcal{K}) \mathrm{MU}$ is split into two parts by a PS-based SWIPT scheme, where $\sqrt{\rho_{k}}$ and $\sqrt{1-\rho_{k}}$ are the 


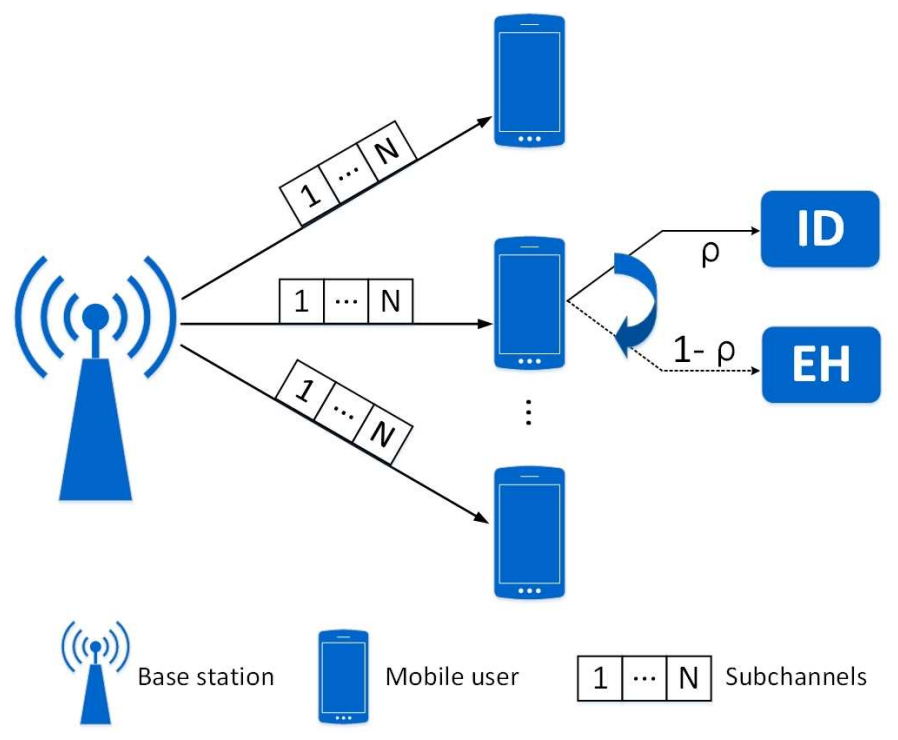

Fig. 1. The system model of the downlink SWIPT-aided MC-NOMA with PS-based receivers.

ratios of the received signal for information decoding (ID) and EH, respectively. Thereby, the received signal for ID and EH can be respectively written as

$$
\begin{gathered}
y_{n, k}^{\mathrm{ID}}=\underbrace{h_{n, k} \sqrt{\rho_{k} p_{n, k}} s_{n, k}}_{\text {Intended signal }}+\underbrace{h_{n, k} \sum_{\substack{j \in \mathcal{K}, j \neq k}} \sqrt{\rho_{k} p_{n, j}} s_{n, j}}_{\text {Interference signal }}+\underbrace{\sqrt{\rho_{k}} z_{n, k}+z_{n, k}^{\mathrm{ID}}}_{\text {Noise }}, \\
y_{n, k}^{\mathrm{EH}}=h_{n, k} \sum_{j=1}^{j=K} \sqrt{\left(1-\rho_{j}\right) p_{n, j}} s_{n, j}+\sqrt{1-\rho_{k}} z_{n, k},
\end{gathered}
$$

in which $z_{n, k}^{\mathrm{ID}} \sim \mathcal{C N}\left(0,\left(\sigma_{n, k}^{\mathrm{ID}}\right)^{2}\right)$ refers to the noise generated during the PS process.

In order to reduce the interference during the ID process, the successive interference cancellation (SIC) technique is applied by the ID receivers. Let $\tilde{h}_{n, k}=h_{n, k}^{2} / \sigma_{n, k}^{2}$ denote the channel to noise ratio (CNR) for the $k$-th MU over the $n$-th SC. In practice, the order of ID in the downlink NOMA is usually the same as the order of the CNR. Therefore, the interference for the $k$-th MU on the $n$-th $\mathrm{SC}$ can be reduced as

$$
I_{n, k}=\rho_{k} h_{n, k}^{2} \sum_{\substack{j \in \mathcal{K}, \tilde{h}_{n, j}>\tilde{h}_{n, k}}} p_{n, j} .
$$


Accordingly, the signal to interference plus noise ratio (SINR) and the available data rate of the $k$-th $\mathrm{MU}$ on the $n$-th $\mathrm{SC}$ can be respectively given by

$$
\begin{gathered}
\operatorname{SINR}_{n, k}=\frac{h_{n, k}^{2} \rho_{k} p_{n, k}}{I_{n, k}+\rho_{k} \sigma_{n, k}^{2}+\left(\sigma_{n, k}^{\mathrm{ID}}\right)^{2}}, \\
R_{n, k}=B W_{n} \log _{2}\left(1+\mathrm{SINR}_{n, k}\right) .
\end{gathered}
$$

Moreover, the harvested energy of the $k$-th MU on the $n$-th SC can be written as

$$
E_{n, k}=\eta\left(1-\rho_{k}\right)\left(h_{n, k}^{2} \sum_{j=1}^{K} p_{n, j}+\sigma_{n, k}^{2}\right),
$$

where $\eta$ corresponds to the efficiency of the EH receivers when harvesting energy.

Hence, the achievable data rate and the available harvested energy for the $k$-th MU can be respectively written as

$$
R_{k}=\sum_{n=1}^{N} R_{n, k}, \quad E_{k}=\sum_{n=1}^{N} E_{n, k} .
$$

Consequently, the total throughput of the considered MC-NOMA system with the application of PS-based SWIPT can be expressed as

$$
R_{\text {sum }}=\sum_{n=1}^{N} \sum_{k=1}^{K} R_{n, k}=\sum_{k=1}^{K} R_{k} .
$$

\subsection{Problem Statement}

In this study, we focus on the total throughput maximization problem for our considered PS-SWIPT aided MC-NOMA system by jointly optimizing the power allocation and splitting control with the constraints of maximum transmit power supply as well as the minimum EH requirement. Thus, the optimization problem can be formulated as follows

$$
\begin{array}{cl}
\max _{\boldsymbol{\rho}, \mathbf{p}} & R_{\text {sum }}(\boldsymbol{\rho}, \mathbf{p}) \\
\text { s.t. } & E_{k} \geq E_{\text {req }}, \quad \forall k \in \mathcal{K}, \\
& 0<\rho_{k}<1, \quad \forall k \in \mathcal{K}, \\
& p_{n, k} \geq 0, \quad \forall n \in \mathcal{N}, \quad \forall k \in \mathcal{K}, \\
& \sum_{n=1}^{N} p_{n, k} \leq p_{k}^{\max }, \quad \forall k \in \mathcal{K},
\end{array}
$$

in which $\boldsymbol{\rho}=\left[\rho_{1}, \rho_{2}, \cdots, \rho_{K}\right]^{T}$ and $\mathbf{p}=\left[\mathbf{p}_{1}, \mathbf{p}_{2}, \cdots, \mathbf{p}_{N}\right]^{T}$ with the component $\mathbf{p}_{n}=\left[p_{n, 1}, p_{n, 2}, \cdots, p_{n, K}\right]^{T}(1 \leq n \leq N)$. The inequality in (11) corresponds to the minimum requirement for $\mathrm{EH}$ of each $\mathrm{MU}$, i.e., $E_{\text {req }} \mathrm{W}$. The inequality in (12) 
indicates that the PS ratio for each MU should be within $(0,1)$. The constraint (13) ensures the non-negativity of the power allocation for the $k$-th MU through the $n$-th SC and the constraint (14) limits the power allocation for the $k$-th MU (i.e., $\left.\sum_{n=1}^{N} p_{n, k}\right)$ not to exceed $p_{k}^{\max }$. Moreover, the maximum power supply of the BS can be implied to be $\sum_{k=1}^{K} P_{k}^{\max }$ according to (14).

The throughput maximization problem formulated in (10)-(14) is non-convex owing to the coupled multiple variables (i.e., $\boldsymbol{\rho}, \mathbf{p}$ ) and the co-channel interference. Additionally, the aforementioned maximization problem is a widely-known NP-hard problem, and hence it is difficult to obtain the solution directly. In the following section, we propose a dual-layer iterative approach to tackle the problem given in (10)-(14).

\section{Algorithm Based on Lagrangian Duality}

In this section, we develop the power allocation and PS control strategy for the involved PS-SWIPT aided MC-NOMA system. Since the coupled variables $\rho$ and p make the original problem (10)-(14) non-convex, it is extremely tough to derive the optimal solution directly. According to [9], for any optimization problem involving multiple variables, it is practicable to deal with the sub-problem over part of variables while considering the remainder as constants, and next turn to handle the sub-problem over the remaining variables. As a result, $\mathbf{p}$ and $\boldsymbol{\rho}$ are separated to develop the practical and effective solution for the considered optimization problem.

\subsection{PS Control With Fixed Power Allocation}

We first consider the case where all the components of the power allocation matrix $\mathbf{p}$ are constants. In this case, we focus on optimizing the PS ratios under the fixed power allocation. Hence, the corresponding sub-problem can be simplified as

$$
\begin{array}{cl}
\max _{\boldsymbol{\rho}} & R_{\mathrm{sum}}(\boldsymbol{\rho}) \\
\text { s.t. } & 0<\rho_{k}<1, \quad \forall k \in \mathcal{K}, \\
& E_{k} \geq E_{\text {req }}, \quad \forall k \in \mathcal{K} .
\end{array}
$$

According to (7), (8) and (17), $\rho_{k}(\forall k \in \mathcal{K})$ is required to satisfy the following condition

$$
\rho_{k} \leq 1-\frac{E_{\mathrm{req}}}{\eta \sum_{n=1}^{N} h_{n, k}^{2} \sum_{j=1}^{K} p_{n, j}+\sigma_{n, k}^{2}} \triangleq \rho_{k}^{\mathrm{UB}} .
$$

Considering (16) and (18) together, the optimization problem (15)-(17) is infeasible unless

$$
\rho_{k}^{\mathrm{UB}}>0, \quad \forall k \in \mathcal{K} .
$$


Proposition 1: Under the fixed power allocation $\overline{\mathbf{p}}$ satisfying (13), (14) and (19), the throughput maximization sub-problem given in (15)-(17) is strictly convex with regard to $\boldsymbol{\rho}$.

Consequently, strong duality holds between the sub-problem (15)-(17) and its corresponding dual problem, which makes it possible to solve (15)-(17) optimally by employing the Lagrangian duality based method [9]. The corresponding Lagrangian function is formulated as

$$
\begin{aligned}
& \mathcal{L}(\boldsymbol{\rho}, \boldsymbol{\mu}, \boldsymbol{\nu}, \boldsymbol{\omega})= \\
& \sum_{n=1}^{N} \sum_{k=1}^{K} B W_{n} \log _{2}\left(1+\frac{\rho_{k} h_{n, k}^{2} \bar{p}_{n, k}}{\rho_{k}\left(h_{n, k}^{2} \sum_{j \in \mathcal{K}, \tilde{h}_{n, j}>\tilde{h}_{n, k}} \bar{p}_{n, j}+\sigma_{n, k}^{2}\right)+C_{n, k}}\right)+\sum_{k=1}^{K} \mu_{k} \rho_{k} \\
& +\sum_{k=1}^{K} \nu_{k}\left(1-\rho_{k}\right)+\sum_{k=1}^{K} \omega_{k}\left(\sum_{n=1}^{N} \eta\left(1-\rho_{k}\right)\left(h_{n, k}^{2} \sum_{j=1}^{K} \bar{p}_{n, j}+\sigma_{n, k}^{2}\right)-E_{\text {req }}\right),
\end{aligned}
$$

in which $\boldsymbol{\mu}=\left[\mu_{1}, \cdots, \mu_{K}\right]^{T}, \boldsymbol{\nu}=\left[\nu_{1}, \cdots, \nu_{K}\right]^{T}$ and $\boldsymbol{\omega}=\left[\omega_{1}, \cdots, \omega_{K}\right]^{T}$ are nonnegative Lagrange multipliers. More specifically, $\boldsymbol{\mu}$ and $\boldsymbol{\omega}$ are corresponding to the constraint (16) while $\boldsymbol{\omega}$ is pertaining to the constraint (17).

Then the Lagrange dual objective function can be accordingly written as

$$
g(\boldsymbol{\mu}, \boldsymbol{\nu}, \boldsymbol{\omega})=\max _{\boldsymbol{\rho}} \mathcal{L}(\boldsymbol{\rho}, \boldsymbol{\mu}, \boldsymbol{\nu}, \boldsymbol{\omega}) .
$$

Thus, the Lagrange dual problem can be modelled as

$$
\begin{aligned}
\min _{\boldsymbol{\mu}, \boldsymbol{\nu}, \boldsymbol{\omega}} & g(\boldsymbol{\mu}, \boldsymbol{\nu}, \boldsymbol{\omega}) \\
\text { s.t. } & \boldsymbol{\mu} \succeq \mathbf{0}, \boldsymbol{\nu} \succeq \mathbf{0}, \boldsymbol{\omega} \succeq \mathbf{0} .
\end{aligned}
$$

To solve the Lagrange dual problem, we first optimize the PS ratio $\rho$ with the given dual variables $\{\boldsymbol{\mu}, \boldsymbol{\nu}, \boldsymbol{\omega}\}$ through gradient ascent method, and then update the dual variables $\{\boldsymbol{\mu}, \boldsymbol{\nu}, \boldsymbol{\omega}\}$ with the optimized $\boldsymbol{\rho}$ through well-known sub-gradient scheme [10].

Optimizing $\rho$ With Given Dual Variables $\{\boldsymbol{\mu}, \boldsymbol{\nu}, \boldsymbol{\omega}\}$ We first calculate the gradient direction of the Lagrangian function (20) regarding the PS ratio $\rho_{k}(\forall k \in \mathcal{K})$, which is given as

$$
\begin{aligned}
\nabla_{\rho_{k}} \mathcal{L} & =\sum_{n=1}^{N} \frac{B W_{n}}{\ln 2} \cdot \frac{A_{n, k} C_{n, k}}{\left(A_{n, k} \rho_{k}+B_{n, k} \rho_{k}+C_{n, k}\right)\left(B_{n, k} \rho_{k}+C_{n, k}\right)} \\
& +\mu_{k}-\nu_{k}-\omega_{k} \sum_{n=1}^{N}\left(\eta h_{n, k}^{2} \sum_{j=1}^{K} \bar{p}_{n, j}+\sigma_{n, k}^{2}\right) .
\end{aligned}
$$

Particularly, $\rho_{k}$ can be sequentially updated according to the following formula

$$
\rho_{k}(n+1)=\rho_{k}(n)+\varepsilon(n) \nabla_{\rho_{k}(n)} \mathcal{L},
$$


where $\rho_{k}(n)$ and $\rho_{k}(n+1)$ denote the $\rho_{k}$ in the $n$-th and $(n+1)$-th iteration respectively, and $\varepsilon(n)$ represents the updating step for $\rho_{k}$ in the $n$-th iteration, which is required to satisfy the following condition

$$
\varepsilon(n)=\left.\arg \max _{\varepsilon} \mathcal{L}(\boldsymbol{\rho}(n+1), \boldsymbol{\mu}, \boldsymbol{\nu}, \boldsymbol{\omega})\right|_{\boldsymbol{\rho}(n+1)=\boldsymbol{\rho}(n)+\varepsilon \nabla_{\boldsymbol{\rho}(n)} \mathcal{L}} .
$$

Process in (25) is repeated until $\left|\nabla_{\rho_{k}(n)} \mathcal{L}\right| \leq \epsilon_{1}$ for any $k \in \mathcal{K}$, and the optimal PS ratio is denoted as $\boldsymbol{\rho}^{*}$. Therefore, the Lagrange dual objective function in (21) is further determined as

$$
g(\boldsymbol{\mu}, \boldsymbol{\nu}, \boldsymbol{\omega})=\mathcal{L}\left(\boldsymbol{\rho}^{*}, \boldsymbol{\mu}, \boldsymbol{\nu}, \boldsymbol{\omega}\right)
$$

Updating $\{\mu, \nu, \omega\}$ With the Optimized $\rho^{*}$ With the obtained PS ratio $\boldsymbol{\rho}^{*}$, the corresponding optimal Lagrange multipliers $\{\boldsymbol{\mu}, \boldsymbol{\nu}, \boldsymbol{\omega}\}$ can be determined accordingly through solving the Lagrange dual problem in (22)-(23).

Obviously, the dual problem is convex on the Lagrange multipliers $\{\boldsymbol{\mu}, \boldsymbol{\nu}, \boldsymbol{\omega}\}$. Therefore, one-dimensional search scheme can be adopted to optimize the dual variables. Nevertheless, the objective function (22) is not necessarily differentiable and thus this gradient-based approach is not always feasible. Otherwise, we apply the widely-used sub-gradient method to determine the dual variables $\{\boldsymbol{\mu}, \boldsymbol{\nu}, \boldsymbol{\omega}\}$, for which the sub-gradient directions are given in Proposition 2.

Proposition 2: The sub-gradient of the Lagrange dual function regarding the Lagrange multipliers can be respectively calculated by

$$
\begin{gathered}
\nabla_{\mu_{k}} g=\rho_{k}^{*} \\
\nabla_{\nu_{k}} g=1-\rho_{k}^{*}, \\
\nabla_{\omega_{k}} g=\sum_{n=1}^{N} \eta\left(1-\rho_{k}^{*}\right)\left(h_{n, k}^{2} \sum_{j=1}^{K} \bar{p}_{n, j}+\sigma_{n, k}^{2}\right)-E_{\text {req }}
\end{gathered}
$$

Proof: Please refer to [10] for more details.

According to Proposition 2, the value of $\mu_{k}\left(\nu_{k}, \omega_{k}\right)$ should decrease if $\nabla_{\mu_{k}} g>$ $0\left(\nabla_{\nu_{k}} g>0, \nabla_{\omega_{k}} g>0\right)$, and vice versa. Based on this observation, we apply the binary search algorithm [10] to determine the optimal Lagrange multipliers (denoted as $\left\{\boldsymbol{\mu}^{*}, \boldsymbol{\nu}^{*}, \boldsymbol{\omega}^{*}\right\}$ ).

The algorithms developed in 1) and 2) operate alternately until the strong duality holds, i.e.,

$$
R_{\text {sum }}\left(\boldsymbol{\rho}^{*}\right)=g\left(\boldsymbol{\mu}^{*}, \boldsymbol{\nu}^{*}, \boldsymbol{\omega}^{*}\right) .
$$

\subsection{Power Allocation With Fixed PS Ratio}

After obtaining the optimal solution of the PS ratio $\rho^{*}$, now we aimed at optimizing the power allocation $\mathbf{p}$ under the optimized $\boldsymbol{\rho}^{*}$. Correspondingly, the original 
optimization problem in (10)-(14) is predigested into the following sub-problem

$$
\begin{aligned}
\max _{\mathbf{p}} & R_{\text {sum }}(\mathbf{p}) \\
\text { s.t. } & E_{k} \geq E_{\text {req }}, \quad \forall k \in \mathcal{K}, \\
& p_{n, k} \geq 0, \quad \forall n \in \mathcal{N}, \quad \forall k \in \mathcal{K}, \\
& \sum_{n=1}^{N} p_{n, k} \leq p_{k}^{\max }, \quad \forall k \in \mathcal{K} .
\end{aligned}
$$

Proposition 3: Suppose that the process of PS in the receiving ends is almost idealized and the noise power for all MUs on the $n$-th SC is equal, i.e., $\left(\sigma_{n, k}^{\mathrm{ID}}\right)^{2} \rightarrow 0$ and $\sigma_{n, k}^{2}=\sigma_{n, j}^{2}=\sigma_{n}^{2}(\forall k, j \in \mathcal{K})$, the sub-problem (32)-(35) is convex if the feasible domain is non-empty.

Similar to the previous section III. $A$, strong duality can also be guaranteed between the sub-problem (32)-(35) and its dual problem, and thus the Lagrangian duality based algorithm is also employed here to optimize the power allocation $\mathbf{p}$.

Specifically, we define the relationship between the $k$-th MU and its decoding order as $k=\pi(i)$. The corresponding Lagrangian function for the sub-problem (32)-(35) can be written as

$$
\begin{aligned}
\widetilde{\mathcal{L}}(\mathbf{p}, \boldsymbol{\alpha}, \boldsymbol{\beta}, \boldsymbol{\gamma}) & =\sum_{n=1}^{N} \sum_{i=1}^{K} B W_{n} \log _{2}\left(1+\frac{h_{n, \pi(i)}^{2} p_{n, \pi(i)}}{h_{n, \pi(i)}^{2} \sum_{j=i+1}^{K} p_{n, \pi(j)}+\sigma_{n}^{2}}\right) \\
& +\sum_{i=1}^{K} \alpha_{i}\left(\sum_{n=1}^{N} \eta\left(1-\rho_{i}^{*}\right)\left(h_{n, \pi(i)}^{2} \sum_{j=1}^{K} p_{n, \pi(j)}+\sigma_{n}^{2}\right)-E_{\text {req }}\right) \\
& +\sum_{n=1}^{N} \sum_{i=1}^{K} \beta_{n, i} p_{n, \pi(i)}+\sum_{i=1}^{K} \gamma_{i}\left(p_{k}^{\max }-\sum_{n=1}^{N} p_{n, \pi(i)}\right)
\end{aligned}
$$

in which $\boldsymbol{\alpha}=\left[\alpha_{1}, \cdots, \alpha_{K}\right]^{T}, \boldsymbol{\beta}=\left[\boldsymbol{\beta}_{1}, \cdots, \boldsymbol{\beta}_{N}\right]^{T}$ with $\boldsymbol{\beta}_{n}=\left[\beta_{n, 1}, \cdots, \beta_{n, K}\right]^{T}$ and $\gamma=\left[\gamma_{1}, \cdots, \gamma_{K}\right]^{T}$ are non-negative multipliers with respect to (33), (34) and (35), respectively.

Then, the Lagrange dual objective function is given by

$$
\widetilde{g}(\boldsymbol{\alpha}, \boldsymbol{\beta}, \boldsymbol{\gamma})=\max _{\mathbf{p}} \widetilde{\mathcal{L}}(\mathbf{p}, \boldsymbol{\alpha}, \boldsymbol{\beta}, \boldsymbol{\gamma}),
$$

Thus, the corresponding dual optimization problem can be formulated as follows

$$
\begin{aligned}
\min _{\boldsymbol{\alpha}, \boldsymbol{\beta}, \boldsymbol{\gamma}} & \widetilde{g}(\boldsymbol{\alpha}, \boldsymbol{\beta}, \boldsymbol{\gamma}) \\
\text { s.t. } & \boldsymbol{\alpha} \succeq \mathbf{0}, \boldsymbol{\beta} \succeq \mathbf{0}, \boldsymbol{\gamma} \succeq \mathbf{0} .
\end{aligned}
$$

The proposed algorithm to solve the aforementioned problems consists of two steps, and more specific details are developed as follows. 
Optimizing $\mathrm{p}$ Under Fixed Lagrange Multipliers $\{\alpha, \beta, \gamma\}$ The gradient ascent method is employed to determine the optimal power allocation $\mathbf{p}^{*}$. Firstly, we analyze the gradient direction of the Lagrangian function given in (36) with regard to the power allocation component $p_{n, \pi(i)}$, which is calculated as

$$
\begin{aligned}
& \nabla_{p_{n, \pi(i)}} \widetilde{\mathcal{L}}=\frac{B W_{n}}{\ln 2} . \\
& \left(\frac{h_{n, \pi(1)}^{2}}{h_{n, \pi(1)}^{2} \Theta_{n, \pi(1)}+\sigma_{n}^{2}}+\sum_{i^{\prime}=2}^{i}\left(\frac{h_{n, \pi\left(i^{\prime}\right)}^{2}}{h_{n, \pi\left(i^{\prime}\right)}^{2} \Theta_{n, \pi\left(i^{\prime}\right)}+\sigma_{n}^{2}}-\frac{h_{n, \pi\left(i^{\prime}-1\right)}^{2}}{h_{n, \pi\left(i^{\prime}-1\right)}^{2} \Theta_{n, \pi\left(i^{\prime}\right)}+\sigma_{n}^{2}}\right)\right) \\
& +\beta_{n, i}-\gamma_{i}+\sum_{j=1}^{K} \alpha_{j} \eta\left(1-\rho_{j}^{*}\right) h_{n, \pi(j)}^{2} .
\end{aligned}
$$

On the $n$-th $(1 \leq n \leq N)$ SC, the power allocation for each MU can be successively updated through the following expressions

$$
\begin{aligned}
\underbrace{p_{n, \pi(1)}(1)-\rightarrow p_{n, \pi(K)}(1)}_{\text {The 1-st iteration }} & \rightarrow \underbrace{p_{n, \pi(1)}(t)-\rightarrow p_{n, \pi(K)}(t)}_{\text {The }(t+1) \text {-th iteration }} \\
& \rightarrow \underbrace{p_{n, \pi(1)}(t+1)--\rightarrow p_{n, \pi(K)}(t+1)}_{\text {Theration }}, \\
p_{n, \pi(i)}(t+1) & =p_{n, \pi(i)}(t)+\widetilde{\varepsilon}(t) \nabla_{p_{n, \pi(i)}(t)} \widetilde{\mathcal{L}}
\end{aligned}
$$

where $t$ and $t+1$ indicate the number of iterations and $\widetilde{\varepsilon}(t)$ represents the updating step in the $t$-th iteration.

The process (41)-(42) for the power allocation on the $n$-th SC proceeds until $\left|\nabla_{p_{n, \pi(i)}} \widetilde{\mathcal{L}}\right| \leq \epsilon_{3}$ for any $1 \leq i \leq K$. Correspondingly, the optimal power allocation on the $n$-th SC is expressed as $p_{n}^{*}$ and thus $\mathbf{p}^{*}=\left[p_{1} *, \cdots, p_{N}^{*}\right]^{T}$. Then, the dual objective function in (37) can be reformulated as

$$
\widetilde{g}(\boldsymbol{\alpha}, \boldsymbol{\beta}, \boldsymbol{\gamma})=\widetilde{\mathcal{L}}\left(\mathbf{p}^{*}, \boldsymbol{\alpha}, \boldsymbol{\beta}, \boldsymbol{\gamma}\right)
$$

Optimizing $\{\boldsymbol{\alpha}, \boldsymbol{\beta}, \boldsymbol{\gamma}\}$ Under the Obtained $\mathrm{p}^{*}$ Similar to the section III. $A$, sub-gradient approach is employed here to tackle the optimization of Lagrange multipliers $\{\boldsymbol{\alpha}, \boldsymbol{\beta}, \boldsymbol{\gamma}\}$, for which the sub-gradient directions are respectively denoted as follows

$$
\begin{gathered}
\nabla_{\alpha_{i}} \widetilde{g}=\sum_{n=1}^{N} \eta\left(1-\rho_{i}^{*}\right)\left(h_{n, \pi(i)}^{2} \sum_{j=1}^{K} p_{n, \pi(j)}+\sigma_{n}^{2}\right)-E_{\mathrm{req}} \\
\nabla_{\beta_{n, i}} \widetilde{g}=p_{n, \pi(i)}
\end{gathered}
$$




$$
\nabla_{\gamma_{i}} \widetilde{g}=p_{k}^{\max }-\sum_{n=1}^{N} p_{n, \pi(i)} .
$$

It is worth noting that the binary search method is also applicable to determine the optimal solution of the Lagrange multipliers here, which are denoted as $\left\{\boldsymbol{\alpha}^{*}, \boldsymbol{\beta}^{*}, \boldsymbol{\gamma}^{*}\right\}$.

The algorithms developed in 1) and 2) are repeated alternately until the zero duality gap is achieved, i.e.,

$$
R_{\mathrm{sum}}\left(\mathbf{p}^{*}\right)=\widetilde{g}\left(\boldsymbol{\alpha}^{*}, \boldsymbol{\beta}^{*}, \boldsymbol{\gamma}^{*}\right) .
$$

\subsection{Complete Solution for Joint Power Allocation and Splitting}

Up to now, the solutions to the sub-problems for optimizing $\boldsymbol{\rho}$ and $\mathbf{p}$ have been proposed in the sections III. $A$ and III. $B$, respectively. Now we develop the complete solution for jointly optimizing the original problem (10)-(14), which is summarized in Algorithm 1.

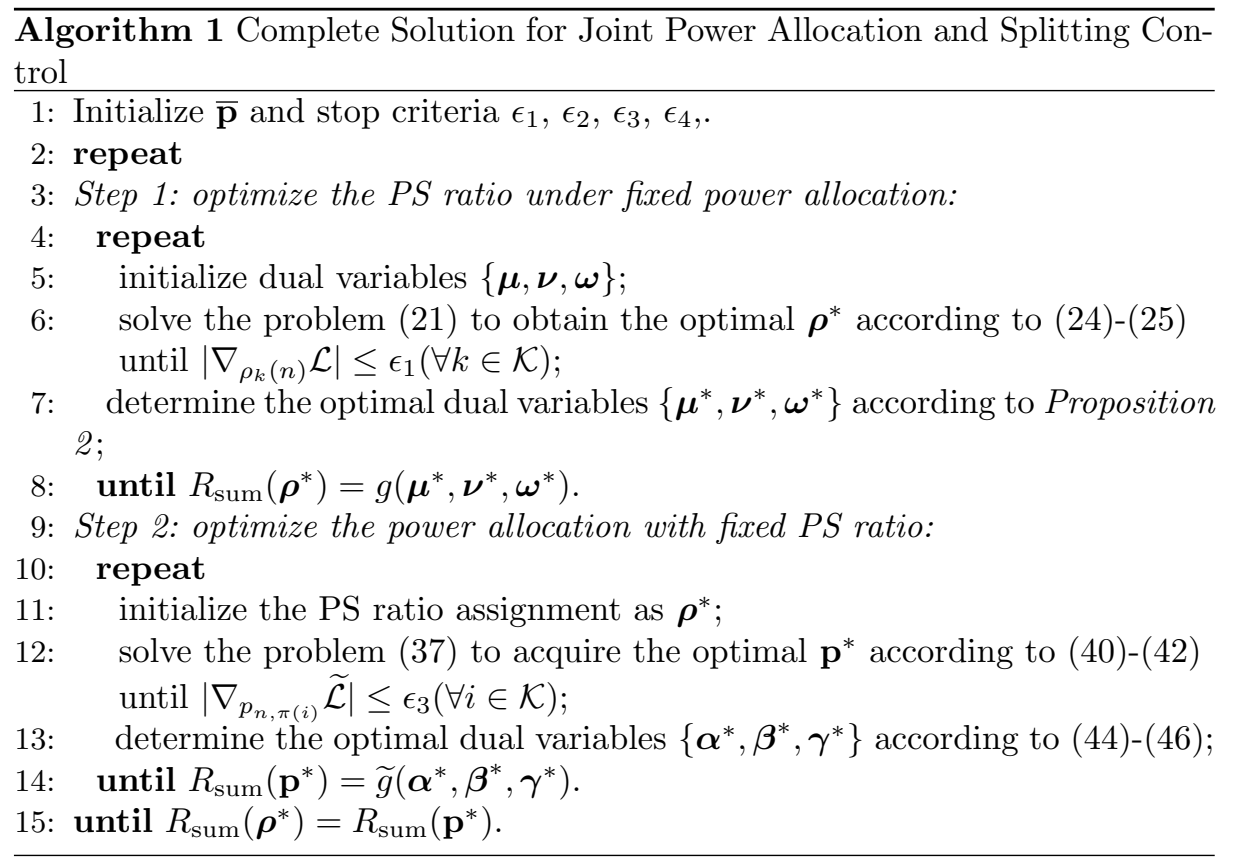

Remark 1: The complete algorithm can be regarded as a dual-layer process. In the inner-layer, the complexity of the gradient decent algorithm is $\mathcal{O}(K)$ and the number of this loop iteration is approximately $\mathcal{O} \log \left(1 / \epsilon_{1}^{2}\right)[11]$; and the complexity of the binary search method with error tolerance $\epsilon_{2}$ is $\mathcal{O} \log \left(1 / \epsilon_{2}^{2}\right)$. Similarly, in the outer-layer, the computational complexity of the 
gradient algorithm method is $\mathcal{O}(N K)$ and the number of this loop iteration is approximately $\mathcal{O} \log \left(1 / \epsilon_{3}^{2}\right)$; and the complexity of the binary search method is $\mathcal{O} \log \left(1 / \epsilon_{4}^{2}\right)$. To summarize, the computational complexity of the complete solution is $\mathcal{O}\left(N K^{2} \log \left(1 / \epsilon_{1}^{2}\right) \log \left(1 / \epsilon_{2}^{2}\right) \log \left(1 / \epsilon_{3}^{2} \log \left(1 / \epsilon_{4}^{2}\right)\right)\right.$.

\section{Numerical Results}

In this section, numerical results are provided to evaluate the convergence performance of our proposed dual-layer iterative approach and the superiority of our considered MC-NOMA system with the application of PS-based SWIPT in terms of throughput. Assume that the BS is located at the center of a circular cell with a radius of $300 \mathrm{~m}$, within which all MUs are randomly and independently located. The available bandwidth of the system is assumed to $B W=100 \mathrm{MHz}$. Referring to the typical 3GPP propagation setting, the channel from the BS to the MU includes three parts, i.e., i.i.d Rayleigh block fading, Log-Normal shadowing with standard deviation of $8 \mathrm{~dB}$ and path loss given by $\left(\frac{d_{0}}{d}\right)^{v}$. In particular, $d, d_{0}=2.5$ and $v=3.76$ indicates the propagation distance, the reference distance and the path-loss exponent, respectively. Moreover, the power spectrum density (PSD) of the channel noise and the additional noise generated during PS process are set to $-96 \mathrm{dBm} / \mathrm{Hz}$ and $-192 \mathrm{dBm} / \mathrm{Hz}$, respectively. The efficiency of the EH circuits is supposed to $\eta=38 \%$.

Firstly, we investigate the convergence performance of the developed duallayer iterative approach. We take a SWIPT-based MC-NOMA system with two SCs and two MUs as an example, where the maximum power supply of the BS and the minimum demand for $\mathrm{EH}$ are set to $4 \mathrm{~W}$ and $0.01 \mathrm{~W}$ respectively. As shown in Fig. 2, it is evident that the proposed Lagrangian duality-based approach is gradually converged to the optimal value acquired by the exhaustive search algorithm. This confirms our convergence analysis.

Then, performance in terms of total throughput of the proposed approach with various constraints is investigated. We taken $N=2, K=2$ and $N=$ $4, K=4$ for comparison. We firstly evaluate the throughput performance under different minimum transmit power supplies. Assume that the minimum requirement for $\mathrm{EH}$ of each $\mathrm{MU}$ is $E_{\text {req }}=0.1 \mathrm{~W}$ and the transmit power budget varies from $2 \mathrm{~W}$ to $20 \mathrm{~W}$. It is obviously shown in Fig. 3 that the total throughput is monotonically non-decreasing with the increase of the transmit power budget. This is because that with the growth in the transmit power budget, the received signal is more likely to be split to ID once the requirement for EH of each MU is satisfied, eventually leading to an increase in the throughput. Then we evaluate the throughput performance under various minimum demands for EH. In particular, it is supposed that the maximum transmit power supply is $10 \mathrm{~W}$ and the minimum requirement for $\mathrm{EH}$ varies from $0.1 \mathrm{~W}$ to $1 \mathrm{~W}$. It is shown in Fig. 4 that the total throughput is monotonically decreasing as the minimum demand for EH grows, resulting from the fact that the received signal is more likely to be split to $\mathrm{EH}$ to fulfill the $\mathrm{EH}$ requirement and thereby the signal split to ID is cut off. Additionally, we can conclude from Fig. 3 and Fig. 4 that our developed 


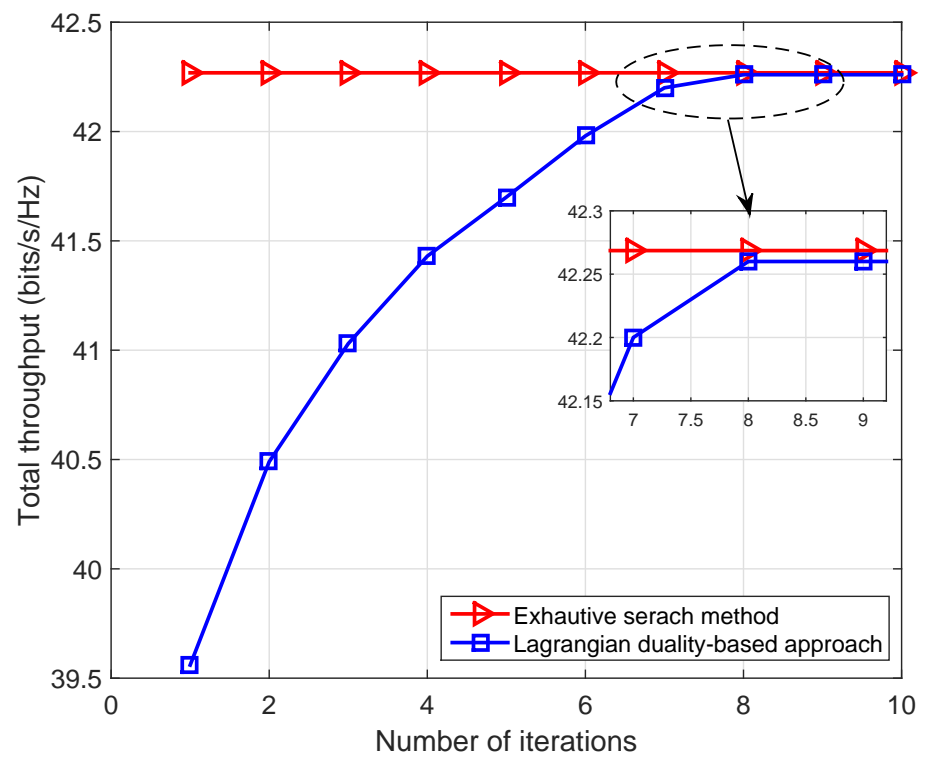

Fig. 2. The convergence behavior of the proposed Lagrangian duality-based approach.

dual-layer iterative approach outperforms the equal power allocation scheme in terms of throughput performance.

Lastly, we examine the performance comparison in terms of total throughput among our considered MC-NOMA with PS-based SIWPT and other schemes in the existing studies, including MC-NOMA with TS-based SWIPT, SC-NOMA with PS-based and OMA with PS-based. In particular, the maximum transmit power supply and the requirement for $\mathrm{EH}$ of each $\mathrm{MU}$ are respectively supposed to $10 \mathrm{~W}$ and $0.1 \mathrm{~W}$, the number of SCs for two MC-NOMA schemes is set to $N=3$, and the number of MUs is assumed to $K=4$. It is evidently depicted in Fig. 5 that both SWIPT-aided MC-NOMA and SWIPT-aided SCNOMA is always superior to the SWIPT-aided OMA, which further confirms that the NOMA scheme is more spectrum-efficient than the conventional OMA scheme. More significantly, our considered PS-based MC-NOMA system outperforms either the TS-based MC-NOMA or the PS-based SC-NOMA. This result demonstrates the superiority of our developed joint power allocation and splitting approach for the considered MC-NOMA system with the application of PS-based SWIPT, and accordingly provides a significant direction for practical communication system design.

\section{Conclusions}

In this work, we have studied the total throughput maximization problem for the downlink MC-NOMA system with the application of PS-based SWIPT un- 


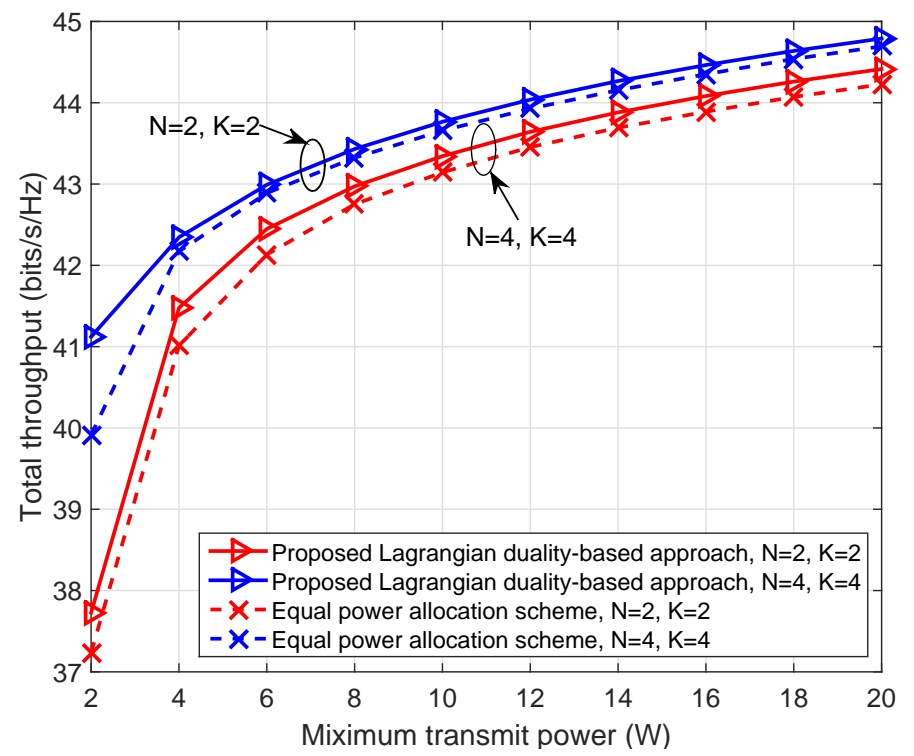

Fig. 3. Throughput performance of the proposed Lagrangian duality-based approach (total throughput vs maximum transmit power).

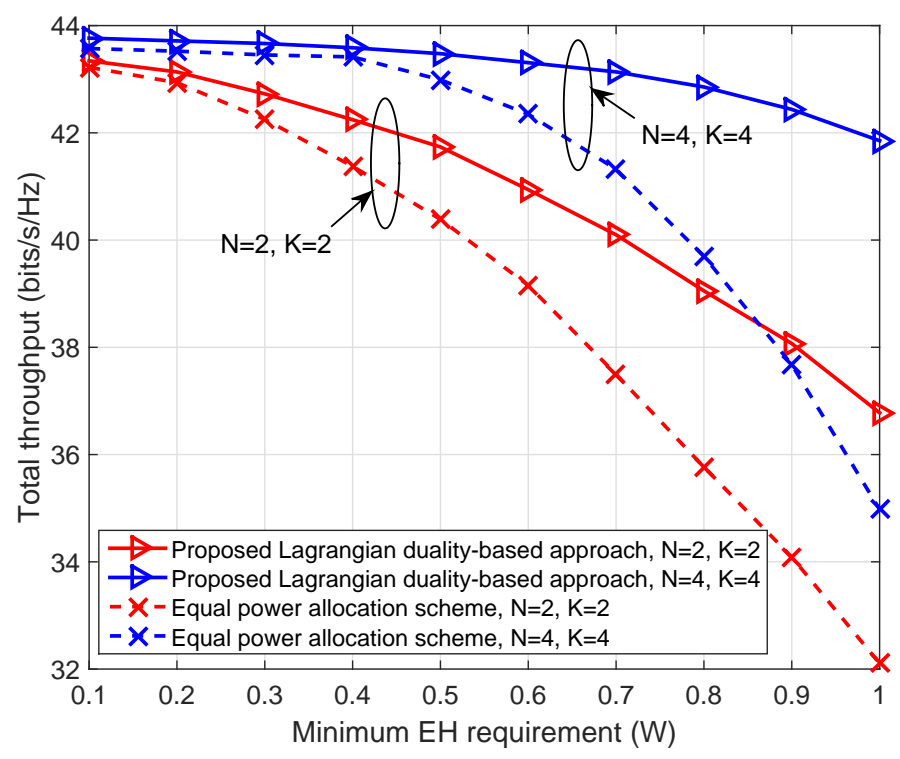

Fig. 4. Throughput performance of the proposed Lagrangian duality-based approach (total throughput vs minimum EH requirement). 


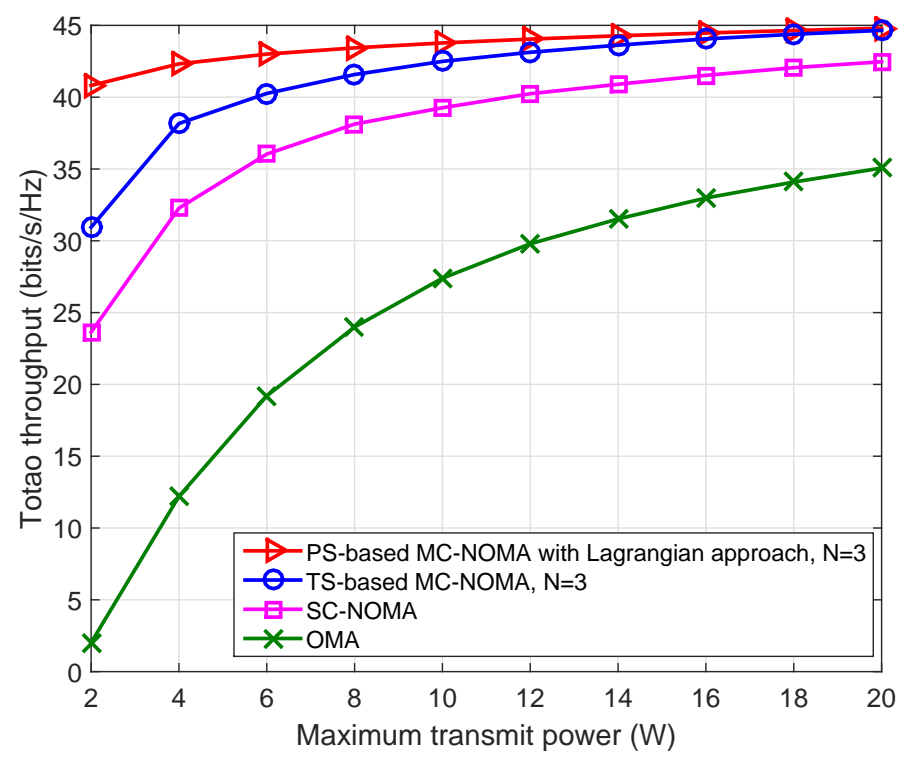

Fig. 5. Performance comparison in terms of throughput among different system model - OMA, SC-NOMA, TS-based MC-NOMA and PS-based MC-NOMA, K=4

der the constraints of the maximum transmit power supply and the minimum demand for EH. The formulated optimization problem was non-convex owing to the coupled variables as well as the multi-user interference, and thus it was challenging to obtain the optimal solution directly. To solve this problem, we proposed a dual-layer iterative approach in which the coupled variables, i.e., the power allocation and the PS ratio assignment, were separated. Then the corresponding sub-problems were solved by employing the Lagrangian dualitybased method. Simulation results verified the theoretical analysis of the convergence performance. More importantly, it was confirmed that the considered MC-NOMA system with the application of PS-based SWIPT outperformed other existing schemes in terms of throughput, including MC-NOMA with TS-SWIPT, SC-NOMA with PS-SWIPT and OMA with PS-SWIPT.

\section{References}

1. A. Benjebbour, Y. Saito, Y. Kishiyama, A. Li, A. Harada, and T. Nakamura. Concept and practical considerations of non-orthogonal multiple access (NOMA) for future radio access. In Proc. IEEE Intell. Signal Process. and Commun. Syst. (IEEE ISPACS), pages 770-774, Nov 2013.

2. Z. Ding, X. Lei, G. K. Karagiannidis, R. Schober, J. Yuan, and V. K. Bhargava. A survey on non-orthogonal multiple access for $5 \mathrm{G}$ networks: Research challenges and future trends. IEEE J. Sel. Areas Commun., 35(10):2181-2195, Oct 2017. 
3. Z. Ding, F. Adachi, and H. V. Poor. The application of MIMO to non-orthogonal multiple access. IEEE Trans. Wireless Commun., 15(1):537-552, Jan 2016.

4. Y. Liu, Z. Ding, M. Elkashlan, and J. Yuan. Nonorthogonal multiple access in largescale underlay cognitive radio networks. IEEE Trans. Veh. Tech., 65(12):1015210157, Dec 2016.

5. Z. Ding, M. Peng, and H. V. Poor. Cooperative non-orthogonal multiple access in 5G systems. IEEE Commun. Lett., 19(8):1462-1465, Aug 2015.

6. L. R. Varshney. Transporting information and energy simultaneously. In Proc. IEEE ISIT, pages 1612-1616, July 2008.

7. J. Tang, J. Luo, M. Liu, D. K. C. So, E. Alsusa, G. Chen, K. Wong, and J. A. Chambers. Energy efficiency optimization for NOMA with SWIPT. IEEE J. Sel. Topics Signal Process., 13(3):452-466, June 2019.

8. Y. Xu, C. Shen, Z. Ding, X. Sun, S. Yan, G. Zhu, and Z. Zhong. Joint beamforming and power-splitting control in downlink cooperative SWIPT NOMA systems. IEEE Trans. Signal Process., 65(18):4874-4886, Sep. 2017.

9. S. Boyd and L. Vandenberghe. Convex Optimization. Cambridge University Press, Cambridge, UK, 2004.

10. L. Zhang, Y. Xin, and Y. Liang. Weighted sum rate optimization for cognitive radio MIMO broadcast channels. IEEE Trans. Wireless Commun., 8(6):2950-2959, June 2009.

11. Jorge J. More Paul H. Calamai. Projected gradient methods for linearly constrained problems. Mathematical Programming, 39(1):93-116, 1987. 